\title{
a-Silicene as oxidation-resistant ultra-thin coating material
}

\author{
Ali Kandemir ${ }^{*}$, Fadil lyikanat ${ }^{2}$, Cihan Bacaksiz ${ }^{2}$ and Hasan Sahin ${ }^{* 3,4}$
}

\section{Full Research Paper}

\section{Address:}

${ }^{1}$ Department of Materials Science and Engineering, Izmir Institute of Technology, 35430, Izmir, Turkey, ${ }^{2}$ Department of Physics, Izmir Institute of Technology, 35430, Izmir, Turkey, ${ }^{3}$ Department of Photonics, Izmir Institute of Technology, 35430, Izmir, Turkey and ${ }^{4}$ ICTP-ECAR Eurasian Center for Advanced Research, Izmir Institute of Technology, 35430, Izmir, Turkey

\section{Email:}

Ali Kandemir ${ }^{*}$ - alikandemir@iyte.edu.tr; Hasan Sahin* -

hasansahin@iyte.edu.tr

* Corresponding author

Keywords:

coating material; density functional theory; oxidization; silicene, silver
Beilstein J. Nanotechnol. 2017, 8, 1808-1814.

doi:10.3762/bjnano.8.182

Received: 11 March 2017

Accepted: 16 August 2017

Published: 31 August 2017

This article is part of the Thematic Series "Silicene, germanene and other group IV 2D materials".

Guest Editor: P. Vogt

(C) 2017 Kandemir et al.; licensee Beilstein-Institut.

License and terms: see end of document.

\section{Abstract}

By performing density functional theory (DFT)-based calculations, the performance of $\alpha$-silicene as oxidation-resistant coating on $\mathrm{Ag}(111)$ surface is investigated. First of all, it is shown that the $\mathrm{Ag}(111)$ surface is quite reactive against $\mathrm{O}$ atoms and $\mathrm{O}_{2}$ molecules. It is known that when single-layer silicene is formed on the $\operatorname{Ag}(111)$ surface, the $3 \times 3$-reconstructed phase, $\alpha$-silicene, is the ground state. Our investigation reveals that as a coating layer, $\alpha$-silicene (i) strongly absorbs single $\mathrm{O}$ atoms and (ii) absorbs $\mathrm{O}_{2}$ molecules by breaking the strong $\mathrm{O}-\mathrm{O}$ bond. (iii) Even the hollow sites, which are found to be most favorable penetration path for oxygens, serves as high-energy oxidation barrier, and (iv) $\alpha$-silicene becomes more protective and less permeable in the presence of absorbed $\mathrm{O}$ atom. It appears that single-layer silicene is a quite promising material for ultra-thin oxidation-protective coating applications.

\section{Introduction}

Surface protection against degradation of a material due to a reaction with its environment has attracted intensive attention of researchers for decades. In order to prevent the loss of important properties (such as conductivity, reflectivity, and mechanical and thermal resistance) of a material, surface protection has been vital nearly for all application areas. As a well-known mechanism of electrochemical corrosion, the formation of rust is an un-solicited reaction between a metal and oxygen. For that reason, protection of surfaces from oxygen has become an important field. Although macroscale and microscale coatings have been used intensively in surface protection for a long time
$[1,2]$, two-dimensional (2D) materials have become new candidates for nanoscale coatings for different material groups. Therefore, coating mechanisms at the nanoscale take are of high interest in nanotechnology, and new candidates for nanostructural protection are needed to be understood in detail.

Due to its extraordinary structural and electrical properties, graphene as 2D material has garnered huge interest in nearly all science branches $[3,4]$. Because of the high impermeability, graphene has also been thought as a corrosion-protection barrier [5-7]. Kirkland et al. investigated the electrochemical response 
of graphene-coated metal surfaces and found that graphene causes reduction in the corrosion rate [8]. In addition, Topsakal et al. showed that graphene is a suitable coating material to protect surfaces from oxidation by performing DFT-based calculations [9]. Bulk forms of transition-metal dichalcogenides (TMDs) are well-known coating materials, and the respective $2 \mathrm{D}$ TMDs can be used as surface protection. In addition, $\mathrm{MoS}_{2}$ is one of the most widely used lubricant coating material [10]. Theoretical and experimental studies have demonstrated that single-layer $\mathrm{MoS}_{2}$ and single layer $\mathrm{W}(\mathrm{S} / \mathrm{Se})_{2}$ can be used as a protective nanocoating material [11-14].

One of the most challenging members of the 2D material family is silicene [15-19], the silicon analogue of graphene. After theoretical prediction [18], silicene was synthesized [19] on a silver surface in the $3 \times 3$-reconstructed $\alpha$-form. Differing from graphene, silicene exhibits low buckling in which atoms in the different sub-lattices are shifted oppositely in the out-of-plane direction. The buckled structure of silicene forms perfect sites to capture oxygen atoms. It is known that silicon atoms tend to bond oxygen atoms strongly and form various stable oxidized silicon structures. Therefore, silicene can be a potential coating material for protection at the nanoscale.

On the other hand, silver is being used in our daily life in jewelry, silverware, decorative objects and electronics. Although the oxidation of silver forms thin layer of $\mathrm{Ag}_{2} \mathrm{O}$, which protects from more oxygen diffusion into silver surface, it is an undesirable reaction. Previous studies have shown that oxidation of silver surfaces leads to increase in work function, color change and significant deterioration of surface quality $[20,21]$. For that reason, coating of silver for protection to oxidation is needed.

In this study, we examined the coating performance of $\alpha$-silicene against oxidation on the most preferable substrate metal for silicene, silver. In first step, we studied the adsorption of an oxygen atom and an oxygen molecule on bare silver with
〈111 growth direction because silicene has been synthesized on $\operatorname{Ag}(111)$ substrates [19]. Then, the adsorption of the oxygen atom/molecule on $\alpha$-silicene over $\operatorname{Ag}(111)$ was investigated. It was shown that $\alpha$-silicene is quite reactive regarding oxidation. In addition, we focused on oxidation scenario of silver in the presence of $\alpha$-silicene. A large energy barrier for oxidation was obtained by performing indentation calculations. In conclusion, it was found silicene exhibits good performance in the protection of a $\operatorname{Ag}(111)$ surface against oxidation.

\section{Results and Discussion Computational methodology}

To obtain the preferable crystal structure of $\alpha$-silicene on $\operatorname{Ag}(111)$, four-layer $\operatorname{Ag}(111)$ composed of two fixed bottom layers and two free upper layers, was prepared as supercell structure with dimensions of $4 \times 4 \times 1$. Thus, the surface of silver successfully simulated with and without silicene on top of $\mathrm{Ag}(111)$. First principles calculations were performed using the Vienna ab initio simulation package (VASP) $[22,23]$, which is based on density functional theory. The projector-augmented wave (PAW) [24,25] formalism was used in the calculations. For the exchange-correlation energy, the generalized gradient approximation of the Perdew-Burke-Ernzerhof (GGA-PBE) [26] functional was used in conjunction with a semi-empirical scheme for including van der Waals (vdW) interaction dispersive forces developed by Grimme [27]. The structural relaxations were performed with a plane wave cut-off energy of at $500 \mathrm{eV}$. A $3 \times 3 \times 1 k$-point mesh was used for the structural relaxation. The criterion of convergence of energy was chosen as $10^{-5} \mathrm{eV}$ between two ionic steps, and the maximum force allowed on each atom is $0.1 \mathrm{meV} / \AA$. At least $13 \AA$ of vacuum were applied along $z$-direction to hinder interactions between the adjacent cells.

\section{Oxidation of the bare Ag surface}

Because the buckled structure of silicene grows sleekly on a silver surface, one may expect unique and enhanced coating
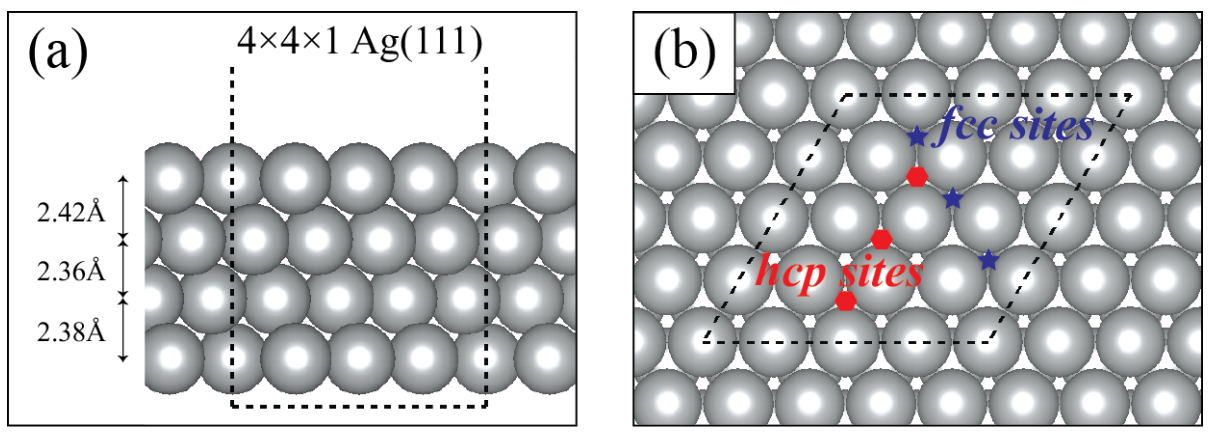

Figure 1: (a) Side view of the $\mathrm{Ag}(111)$ supercell structure and (b) top view of the $\mathrm{Ag}(111)$ supercell structure with possible oxygen captured sites Definitions and oxygen binding energies of all sites are given in Table 1. 
performance against oxidation. As shown in Figure 1, we first investigate how strongly an oxygen atom and an oxygen molecule interact with possible sites on the $\operatorname{Ag}(111)$ surface. Two sites were found to be preferable locations for oxygen atoms on the $\operatorname{Ag}(111)$ surface, the hcp and fcc hollow sites. These sites are shown in Figure 1b.

Figure 2a and Figure 2b show how an oxygen atom is adsorbed at the silver surface at the hcp and fcc hollow sites, respectively. The binding energy of single oxygen on the $\operatorname{Ag}(111)$ surface is about $3.9 \mathrm{eV}$. The energy difference between sites is only $110 \mathrm{meV}$. It is seen that silver surface strongly captures single oxygen atoms. Oxygen in fcc site forms a bond distance with neighbor silver atoms of about $2.14 \AA$. The presence of oxygen causes a distortion of about $9.8 \%$ and pushes neighbor atoms in this site. Although oxygen at a hep site has the same bond distance with neighbor atoms as at the fcc site, the presence of
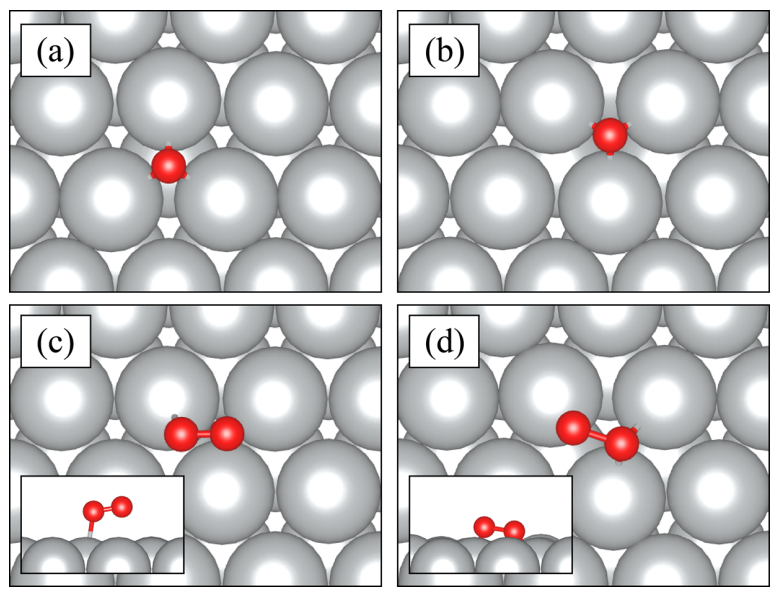

Figure 2: Top view of $\mathrm{Ag}(111)$ geometric structures after capturing an oxygen atom and an $\mathrm{O}_{2}$ molecule. (a) hcp hollow site and (b) fcc hollow site for oxygen, (c) magnetic and (d) nonmagnetic $\mathrm{O}_{2}$ molecule adsorption on $\mathrm{Ag}(111)$. The insets in panel (c) and panel (d) show the bonding characteristics of $\mathrm{O}_{2}$ molecule.

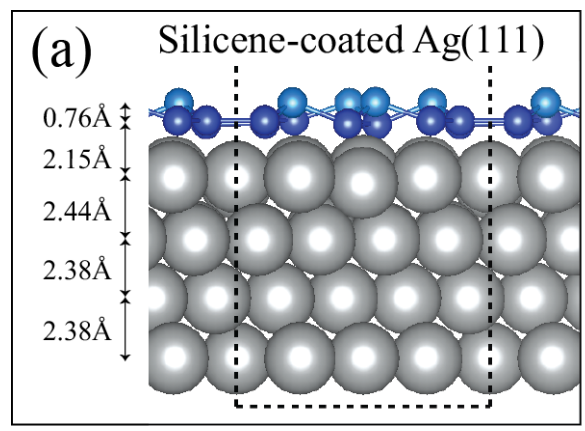

oxygen causes distortion of about $8.9 \%$ at the hep site. It appears that, absence of the lower silver atom at the fcc site results in a deeper penetration of the oxygen atom than at the hcp site.

Compared to a single oxygen atom, the $\mathrm{O}_{2}$ molecule behaves differently on a bare silver surface. Figure $2 \mathrm{c}$ and Figure 2d) show how the $\mathrm{O}_{2}$ molecule interacts with the silver surface. Magnetic and nonmagnetic states of $\mathrm{O}_{2}$ molecule are observed while oxygen is captured at the silver surface. In the magnetic state, only one of the oxygens come closer to the surface. In the other case, both oxygen atoms come closer to the surface. In both cases, the binding energies are ca. $200 \mathrm{meV}$. Therefore, it is seen that $\mathrm{O}_{2}$ molecule tends to bind to the silver surface. In the magnetic state, the oxygen-oxygen distance is around $1.26 \AA$, which is close to oxygen-oxygen distance in an oxygen molecule in the vacuum state. In the nonmagnetic state, the oxygen-oxygen distance is around $1.41 \AA$. The silver surface weakens the oxygen-oxygen bond in the nonmagnetic system. In addition, the distance between oxygen and silver is nearly the same as the distance between a single oxygen atom and silver distance, which is $2.14 \AA$. Similar to adsorption of a single atom, the oxygen molecule distorts silver surface at that site. Therefore, the oxygen-oxygen bond can be broken through a thermally induced process and the silver surface can exhibit oxygen atoms.

\section{Oxidation of the silicene-coated Ag surface}

In this section, the silicene-coated $\operatorname{Ag}(111)$ surface is investigated. We consider the experimentally realized structure of silicene on $\operatorname{Ag}(111), \alpha$-silicene [19]. Albeit with a different notation, it was also shown that $\alpha$-silicene is the thermodynamically favorable phase under a wide range of conditions [17]. Differing from the theoretical predicted simply buckled silicene, $\alpha$-silicene has a $3 \times 3$-reconstruction in which six silicon atoms form a sub-layer over the other twelve silicon atoms in the supercell as shown in Figure 3. Blue and turquoise atoms repre-

Figure 3: (a) Side view of the silicene-coated $\mathrm{Ag}(111)$ supercell structure and (b) top view of the silicene-coated $\mathrm{Ag}(111)$ supercell structure with possible oxygen-capture sites. Definitions and oxygen binding energies of all sites are given in Table 1. 
sent the lower and upper $\mathrm{Si}$ atoms respectively. In the presence of silicene, the interlayer distance of the uppermost Ag layers are slightly changing and the distance between Ag surface and silicene is found to be ca. $2.15 \AA$.

In Figure 3b, possible sites for oxygen on silicene-coated $\operatorname{Ag}(111)$ are demonstrated. There are six possible sites due to symmetry in silicene-coated $\operatorname{Ag}(111)$. These sites are named according to hollow and top sites of neighboring silicon atoms in the buckled silicene structure. $\mathrm{H}_{x} \mathrm{~s}$ are for hollow sites, $\mathrm{T}_{x} \mathrm{~s}$ are for top sites. The definition of sites can be found in Table 1 in detail. These six possible sites reflect all possible final configurations in the system. Table 1 shows the oxygen binding energy of all possible sites for silicene-coated $\operatorname{Ag}(111)$.

Silicene captures oxygen with a binding energy range of 5.8-6.5 eV. Compared to silver, silicene has a higher tendency to bind to oxygen. Figure 4 shows the oxygen-silicene bonding characteristics when the system reaches its local lowest energy. The least preferable sites for oxygen on silicene-coated $\mathrm{Ag}(111)$ are the sites where the highest distortion occurs and where oxygen has a low interaction with silicon atoms, namely $\mathrm{H}_{2}, \mathrm{H}_{3}$ and $\mathrm{T}_{1}$. On the other hand, the sites with the lowest distortion are the most preferable sites for oxygen on silicene-coated $\operatorname{Ag}(111)$, namely $\mathrm{T}_{2}, \mathrm{H}_{1}$ and $\mathrm{T}_{3}$ in the order from the lowest to the highest binding energy. The final configurations of $\mathrm{T}_{2}$ and $\mathrm{H}_{1}$ with oxygen presence seem to be equivalent. Oxygen binds to a lower silicon atoms, but the difference lies in the fact that the upper silicon atoms in $\mathrm{T}_{2}$ allow for more freedom and oxygen can take a position between them. Whereas, six lower silicon atoms tend to retain their hexagonal configuration and the oxygen atom finds itself in an upper-site position between two silicon atoms. The absence of a silicon-silicon bond in the $\mathrm{T}_{2}$ configuration results in a difference of $180 \mathrm{meV}$. The highest

\begin{tabular}{|c|c|c|c|c|}
\hline system & site & name & binding energy (eV) & $\Delta_{\mathrm{BE}}(\mathrm{meV})$ \\
\hline \multirow[t]{2}{*}{ O@Ag } & fcc & cubic close-packed hollow site & 3.90 & - \\
\hline & hcp & hexagonal close-packed hollow site & 3.79 & 110 \\
\hline \multirow[t]{6}{*}{ O@Si/Ag } & $\mathrm{H}_{1}$ & site between six lower silicon atoms & 6.45 & 70 \\
\hline & $\mathrm{T}_{1}$ & top site of upper silicon atom & 5.78 & 740 \\
\hline & $\mathrm{H}_{2}$ & site between three upper and three lower silicon atoms & 6.00 & 520 \\
\hline & $\mathrm{T}_{2}$ & top site between two lower silicon atoms & 6.27 & 250 \\
\hline & $\mathrm{T}_{3}$ & top site between one upper and one lower silicon atom & 6.52 & - \\
\hline & $\mathrm{H}_{3}$ & site between four lower and two upper silicon atoms & 5.84 & 680 \\
\hline \multirow[t]{2}{*}{$\mathrm{O}_{2} @ \mathrm{Ag}$} & mag & magnetic $\mathrm{O}_{2}$ molecule on the surface & 0.21 & - \\
\hline & n-mag & non-magnetic $\mathrm{O}_{2}$ molecule on the surface & 0.19 & 20 \\
\hline \multirow[t]{2}{*}{$\mathrm{O}_{2} @ \mathrm{Si} / \mathrm{Ag}$} & in-S & $\mathrm{O}_{2}$ molecule inside silicene & 6.70 & - \\
\hline & top-S & $\mathrm{O}_{2}$ molecule on top of silicene & 2.06 & 4640 \\
\hline
\end{tabular}
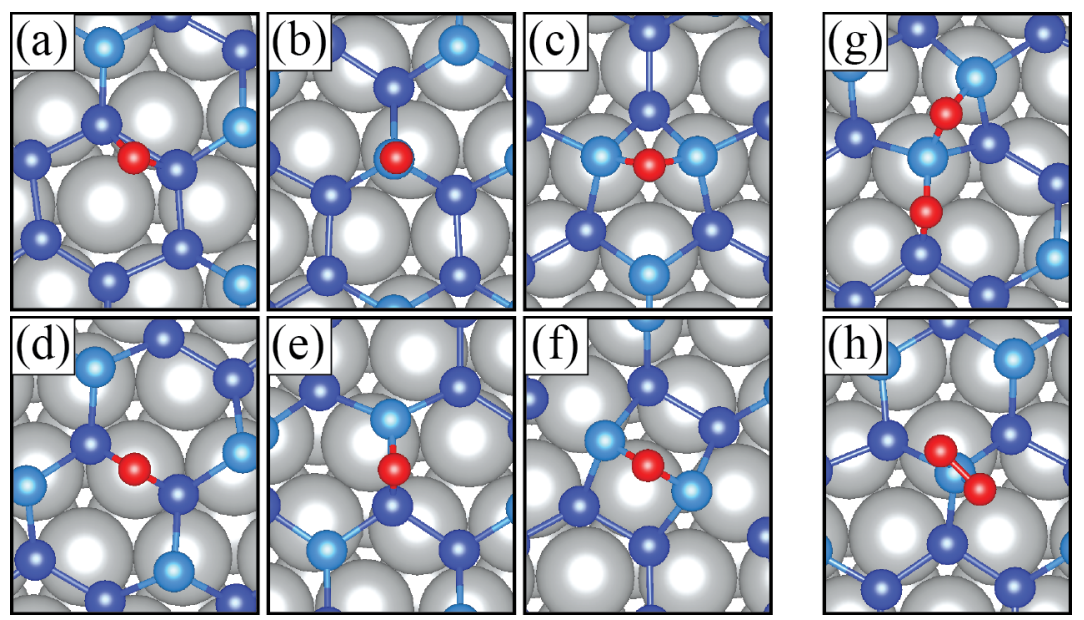

Figure 4: Final configurations of silicene-coated $A g\left(111\right.$ ) after capturing an oxygen atom at the sites (a) $H_{1}$, (b) $T_{1}$, (c) $H_{2}$, (d) $T_{2}$, (e) $T_{3}$ and (f) $H_{3}$. Final configurations $(\mathrm{g})$ in-S and $(\mathrm{h})$ top-S of silicene-coated $\mathrm{Ag}(111)$ after capturing an $\mathrm{O}_{2}$ molecule. 
binding energy is calculated for $\mathrm{T}_{3}$ site. In this configuration, the oxygen atom enters a top-site position between upper and a lower silicon atoms. In this position, the lowest distortion occurs in the system so that oxygen binds with a high energy and leads to formation of an energetically favorable final configuration.

In the case of the $\mathrm{O}_{2}$ molecule, there are two final configurations, "in-S" and "top-S", to be formed at the most favorable oxygen-capture sites. Figure $4 \mathrm{~g}$ and Figure $4 \mathrm{~h}$ show the in-S and top-S configurations, respectively. It is shown that silicon and oxygen strongly interact with each other (Table 1 ). $\mathrm{SiO}_{2}$ is one of the most stable compounds in nature. Therefore, silicon tends to bind two oxygen atoms in the form of $\mathrm{SiO}_{2}$ as depicted in Figure $4 \mathrm{~h}$. However, the energetically favorable configuration is the one in which oxygen atoms take separate positions between the silicon atoms (Figure $4 \mathrm{~g}$ ). In contrast to the other configuration, the silicon atoms in silicene can pluck the $\mathrm{O}_{2}$ molecule and are allowed to diffuse in the system. This promises a good oxidation barrier for the silver surface.

\section{Oxidation of the Ag surface in the presence of silicene coating}

To investigate the application of silicene as an oxidation barrier, indentation calculations with oxygen were performed. Since O atoms and $\mathrm{O}_{2}$ molecules strongly interact with silicene, the diffusion of $\mathrm{O} / \mathrm{O}_{2}$ in the lateral direction is not possible and the hollow sites of the hexagonal lattice are the only possible sites for the penetration into the structure. Therefore, the hollow sites are considered for the indentation simulation. There are four different hollow sites in the silicene structure on $\operatorname{Ag}(111)$, as shown in Figure $3 b$. The sites are denoted as $\mathrm{H}_{1}, \mathrm{H}_{2}, \mathrm{H}_{2}$ ' and $\mathrm{H}_{3} . \mathrm{H}_{2}$ and $\mathrm{H}_{2}$ ' sites coincide to the fcc and hep sites of the $\operatorname{Ag}(111)$ surface, respectively.

For the indentation calculations, a single oxygen atom is placed in the middle of a hollow site. Calculations are performed as follows: First, the oxygen atom is kept at a distance of about $10 \AA$ from the Ag surface (ca. $8 \AA$ to the silicene surface) and is approached to the surface in $1.0 \AA$ steps. When the oxygen atom interacts with silicene, the step size is reduced to $0.5 \AA$. In Figure 5, the change of the total energy as a function of the vertical distance between oxygen atom and Ag surface is given for the different hollow sites. The barrier energies $\left(E_{\mathrm{br}}\right)$, which are defined as the amount of energy needed for an oxygen atom to pass through a hollow site, are also shown in Figure 5. From lowest to highest, the values of $E_{\mathrm{br}}$ for $\mathrm{H}_{1}, \mathrm{H}_{2}, \mathrm{H}_{2}$ ' and $\mathrm{H}_{3}$ are found to be 1.66, 1.82, 1.85 and $1.99 \mathrm{eV}$, respectively. The $\mathrm{H}_{3}$ hollow site has the highest energy barrier among all sites. At first, a fixed single oxygen atom attracts two upper silicon atoms to form one of the highly stable configurations. For that reason, the lowest ground-state energy occurs in this hollow site. While the single oxygen atom approaches the Ag surface, a barrier occurs up to ca. $2.5 \AA$ where oxygen passes through hollow site exactly from upper site of silicene to the lower site of silicene. As seen in Figure 5, this also happens at the $\mathrm{H}_{2}, \mathrm{H}_{2}$, and $\mathrm{H}_{3}$ hollow sites. $\mathrm{H}_{2}$ and $\mathrm{H}_{2}$ ' are similar hollow sites. There is a small difference, ca. $30 \mathrm{meV}$, in energy barrier at these hollow sites. The lowest energy barrier, $1.66 \mathrm{eV}$, is seen in the $\mathrm{H}_{1}$ hollow site. Due to the planar structure of the silicon atoms at the $\mathrm{H}_{1}$ site, it is the most suitable hollow site for an oxygen atom to pass through silicene. Since the $\mathrm{H}_{1}$ hollow site has the lowest barrier energy, the side view of the structure for different distances is shown in the inset of Figure 5. At about $5 \AA$, the single oxygen atom pulls and binds one of the lower silicon atoms. Because further penetration of oxygen is not favorable at that distance from the Ag surface, a small local minimum occurs. Then, the other lower silicon atom binds the oxygen and the energy is lowered further. Due to the larger void size in the $\mathrm{H}_{1}$ hollow site than in the other hollow sites, it is easy to pass for the oxygen atom from the upper surface of silicene to the lower surface. At a distance of around $2.0 \AA$, the barrier has a different value than at the other hollow sites, because of the planar formation of the lower silicon atoms and also because of the larger void of the $\mathrm{H}_{1}$ hollow site.

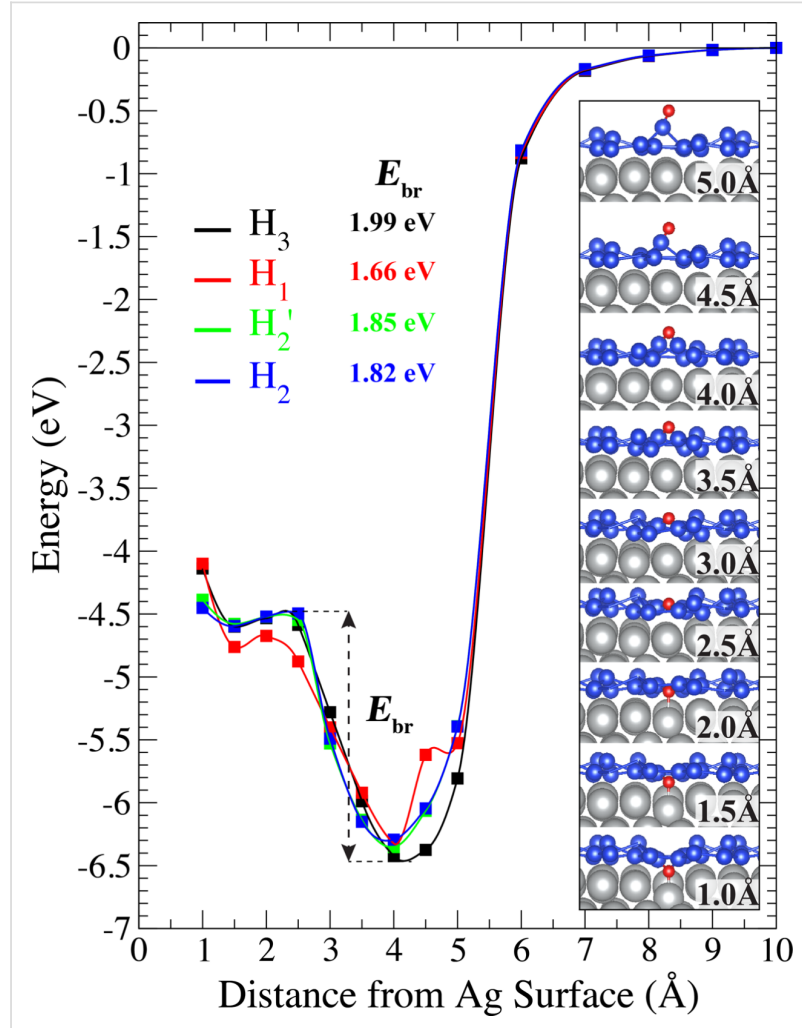

Figure 5: Indentation of an oxygen atom at different hollow sites. $E_{\mathrm{br}}$ is the energy barrier the oxygen atom needs to pass inside the silicene environment. Inset graph shows the oxygen progression in $\mathrm{H}_{1}$. 
$\mathrm{H}_{1}$ is more permeable for oxygen atoms than other hollow sites. Hence, the indentation calculations for the $\mathrm{O}_{2}$ molecule are performed at the $\mathrm{H}_{1}$ site. One oxygen atom of the $\mathrm{O}_{2}$ molecule is fixed, the other non-restricted oxygen follows the fixed oxygen naturally. The oxygen molecule is moved closer to the Ag surface step by step from a distance of $10 \AA$ via the fixed oxygen atom. The indentation process is shown with a small step size after the oxygen atom is captured by a silicon atom (Figure 6). There is a local-minimum state in which oxygen is still in the molecule form (see inset graphs in Figure 6), far from the Ag surface at a distance of about $4.5 \AA$. While the indentation of the $\mathrm{O}_{2}$ molecule continues, the energy increases up to some point. At distances below $3.8 \AA$, a sudden decrease in energy is seen. The inset graphs in Figure 6 show that the deterioration of the $\mathrm{O}_{2}$ molecule happens due to a strong interaction between oxygen and silicon atoms and after that silicene-coated silver system reaches a global-minimum state. The figure shows that the dissociation of the oxygen molecule is favorable and requires ca. $300 \mathrm{meV}$ to exceed the energy barrier of transition. Since the $\mathrm{H}_{1}$ hollow site is the most permeable site, the maximum value of the transition energy barrier converges to ca. $300 \mathrm{meV}$. Two possible configurations, in-S and top-S (Figure $4 \mathrm{~g}, \mathrm{~h}$ ), are observed. The difference of those two

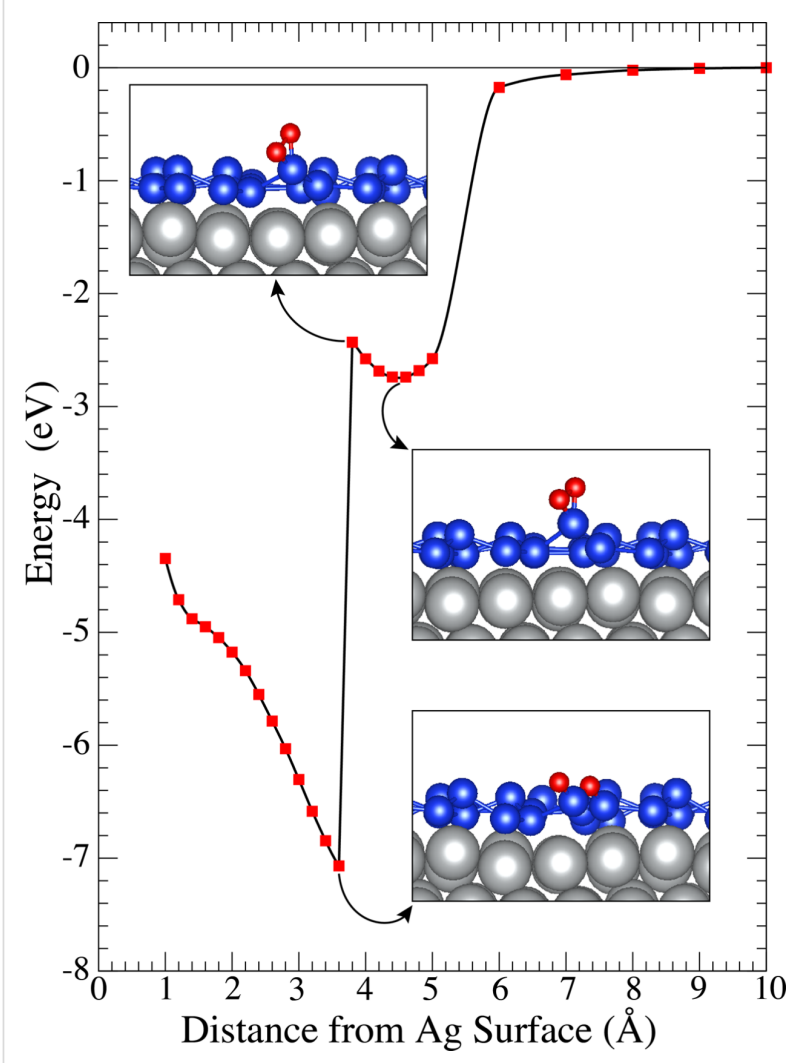

Figure 6: Indentation calculations for an $\mathrm{O}_{2}$ molecule. Inset graphs show structures according to local-minimum states of $\mathrm{O}_{2}$, before and after reaching the global minimum state of the system. minimum-energy states is similar (Table 1). In addition, oxidized silicene does not allow for a further indentation of a single oxygen atom. The locally forming silicon oxide structure attracts oxygen atoms more strongly. A local-minimum state as in FigureFigure 5 is not found between silicene and the Ag surface. Therefore, silicene becomes less permeable and more protective with increasing number of oxygen atoms. Our findings are consistent with the recent studies of oxidized silicene $[28,29]$, which observed non-oxidized metal surfaces after the oxidation of silicene on metal substrates. One may claim that silicene retains its extreme reactivity to oxygen atoms even after forming localized silicon-oxide structures. As a result, silicene has great potential to capture unwanted atoms and to protect the metal surface.

\section{Conclusions}

In this study, we performed first principles calculations to investigate the oxidation properties of $\alpha$-silicene as a coating material on $\mathrm{Ag}(111)$. It was found that an $\mathrm{O}_{2}$ molecule interact with the Ag surface with a low binding energy, while a single oxygen atom interact strongly with the surface. The silicene coating on Ag surface was demonstrated as protective material from oxidation. In particular, large binding energies between a single oxygen atom and silicene were calculated for the possible adsorption sites. This strong interaction can break the oxygen-oxygen bond as well. Moreover, the energy barriers for the oxygen atom between silicene and Ag surface are quite high and sufficient for the protection of the metal surface. Indentation calculations of the $\mathrm{O}_{2}$ molecule showed that the molecule dissociates in the vicinity of silicene. It is also seen that an increase in oxygen atoms makes silicene more protective and silicene does not allow oxygen to pass to the metal surface. In conclusion, silicene has been proven itself as oxidation-resistant nanocoating material.

\section{Acknowledgements}

The calculations were performed at TUBITAK ULAKBIM, High Performance and Grid Computing Center (TR-Grid e-Infrastructure). H.S. acknowledges financial support from the TUBITAK under the project number 116C073. H.S. acknowledges support from Bilim Akademisi-The Science Academy, Turkey under the BAGEP program.

\section{References}

1. Sørensen, P. A.; Kiil, S.; Dam-Johansen, K.; Weinell, C. E. J. Coat. Technol. Res. 2009, 6, 135-176. doi:10.1007/s11998-008-9144-2

2. Gray, J. E.; Luan, B. J. Alloys Compd. 2002, 336, 88. doi:10.1016/S0925-8388(01)01899-0

3. Novoselov, K. S.; Geim, A. K.; Morozov, S. V.; Jiang, D.; Zhang, Y.; Dubonos, S. V.; Grigorieva, I. V.; Firsov, A. A. Science 2004, 306, 666-669. doi:10.1126/science.1102896 
4. Geim, A. K.; Novoselov, K. S. Nat. Mater. 2007, 6, 183-191. doi:10.1038/nmat1849

5. Chen, S.; Brown, L.; Levendorf, M.; Cai, W.; Ju, S.-Y.; Edgeworth, J.; Li, X.; Magnuson, C. W.; Velamakanni, A.; Piner, R. D.; Kang, J.; Park, J.; Ruoff, R. S. ACS Nano 2011, 5, 1321-1327. doi:10.1021/nn103028d

6. Bunch, J. S.; Verbridge, S. S.; Alden, J. S.; van der Zande, A. M.; Parpia, J. M.; Craighead, H. G.; McEuen, P. L. Nano Lett. 2008, 8, 2458-2462. doi:10.1021/nl801457b

7. Böhm, S. Nat. Nanotechnol. 2014, 9, 741-742. doi:10.1038/nnano.2014.220

8. Kirkland, N. T.; Schiller, T.; Medhekar, N.; Birbilis, N. Corros. Sci. 2012, 56, 1. doi:10.1016/j.corsci.2011.12.003

9. Topsakal, M.; Şahin, H.; Ciraci, S. Phys. Rev. B 2012, 85, 155445. doi:10.1103/PhysRevB.85.155445

10. Winer, W. O. Wear 1967, 10, 422. doi:10.1016/0043-1648(67)90187-1

11.Sen, H. S.; Sahin, H.; Peeters, F. M.; Durgun, E. J. Appl. Phys. 2014, 116, 083508. doi:10.1063/1.4893790

12. Santosh, K. C.; Longo, R. C.; Wallace, R. M.; Cho, K. J. Appl. Phys. 2015, 117, 135301. doi:10.1063/1.4916536

13. Dixit, S.; Popat, P. P.; Rawat, S. S.; Sivarajan, S. Indian J. Sci. Technol. 2016, 9.

14. Domínguez-Meister, S.; Rojas, T. C.; Brizuela, M.; Sánchez-López, J. C. Sci. Technol. Adv. Mater. 2017, 18, 122-133. doi:10.1080/14686996.2016.1275784

15. Zhao, J.; Liu, H.; Yu, Z.; Quhe, R.; Zhou, S.; Wang, Y.; Liu, C. C.; Zhong, H.; Han, N.; Lu, J.; Yao, Y.; Wu, K. Prog. Mater. Sci. 2016, 83, 24. doi:10.1016/j.pmatsci.2016.04.001

16. Liu, H.; Feng, H.; Du, Y.; Chen, J.; Wu, K.; Zhao, J. 2D Mater. 2016, 3 , 025034. doi:10.1088/2053-1583/3/2/025034

17. Liu, H.; Han, N.; Zhao, J. Appl. Surf. Sci. 2017, 409, 97. doi:10.1016/j.apsusc.2017.03.007

18. Cahangirov, S.; Topsakal, M.; Aktürk, E.; Şahin, H.; Ciraci, S. Phys. Rev. Lett. 2009, 102, 236804. doi:10.1103/PhysRevLett.102.236804

19. Vogt, P.; De Padova, P.; Quaresima, C.; Avila, J.; Frantzeskakis, E.; Asensio, M. C.; Resta, A.; Ealet, B.; Le Lay, G. Phys. Rev. Lett. 2012, 108, 155501. doi:10.1103/PhysRevLett.108.155501

20. Rehren, C.; Muhler, M.; Bao, X.; Schlögl, R.; Ertl, G. Z. Phys. Chem. 1991, 174, 11. doi:10.1524/zpch.1991.174.Part_1.011

21. Chen, Z. Y.; Liang, D.; Ma, G.; Frankel, G. S.; Allen, H. C.; Kelly, R. G. Corros. Eng., Sci. Technol. 2010, 45, 169. doi:10.1179/147842209X12579401586681

22. Kresse, G.; Hafner, J. Phys. Rev. B 1993, 47, 558. doi:10.1103/PhysRevB.47.558

23. Kresse, G.; Furthmüller, J. Phys. Rev. B 1996, 54, 11169-11186. doi:10.1103/PhysRevB.54.11169

24. Kresse, G.; Joubert, D. Phys. Rev. B 1999, 59, 1758-1775. doi:10.1103/PhysRevB.59.1758

25. Blöchl, P. E. Phys. Rev. B 1994, 50, 17953-17979. doi:10.1103/PhysRevB.50.17953

26. Perdew, J. P.; Burke, K.; Ernzerhof, M. Phys. Rev. Lett. 1996, 77, 3865-3868. doi:10.1103/PhysRevLett.77.3865

27. Grimme, S. J. Comput. Chem. 2006, 27, 1787-1799. doi:10.1002/jcc.20495

28. Du, Y.; Zhuang, J.; Liu, H.; Xu, X.; Eilers, S.; Wu, K.; Cheng, P.; Zhao, J.; Pi, X.; See, K. W.; Peleckis, G.; Wang, X.; Dou, S. X. ACS Nano 2014, 8, 10019-10025. doi:10.1021/nn504451t
29. Du, Y.; Zhuang, J.; Wang, J.; Li, Z.; Liu, H.; Zhao, J.; Xu, X.; Feng, H.; Chen, L.; Wu, K.; Wang, X.; Dou, S. X. Sci. Adv. 2016, 2, e1600067. doi:10.1126/sciadv.1600067

\section{License and Terms}

This is an Open Access article under the terms of the Creative Commons Attribution License

(http://creativecommons.org/licenses/by/4.0), which permits unrestricted use, distribution, and reproduction in any medium, provided the original work is properly cited.

The license is subject to the Beilstein Journal of

Nanotechnology terms and conditions:

(http://www.beilstein-journals.org/bjnano)

The definitive version of this article is the electronic one which can be found at:

doi:10.3762/bjnano.8.182 